\title{
Description of Transport Codes for Space Radiation Shielding
}

Myung-Hee Y. Kim¹, John W. Wilson², and Francis A. Cucinotta ${ }^{3}$

${ }^{1}$ Division of Space Life Sciences, Universities Space Research Association, Houston, TX 77058 ${ }^{2}$ Distinguished Research Associates, NASA Langley Research Center, Hampton, VA 23681

${ }^{3}$ Space Radiation Program, NASA Johnson Space Center, Houston, TX 77058 


\section{Introduction}

- Radiation transport codes, when combined with Risk Projection models, are main tool for shielding study and design.

- Approaches to assess the accuracy of Transport Codes:

- Ground-based studies with defined beams and material layouts

- Inter-comparison of transport code results for matched boundary conditions

- Comparisons to flight measurements

- NASA's HZETRN/QMSFRG code has a very high degree of congruence for each of these criteria. 


\section{Components of Space Radiation Shield Design}

\begin{tabular}{|l|}
\hline Environmental Models \\
- Trapped Radiations \\
- Solar Energetic Particles \\
- Galactic Cosmic Rays \\
- Laboratory lon Beams
\end{tabular}

$\downarrow$ External Environment

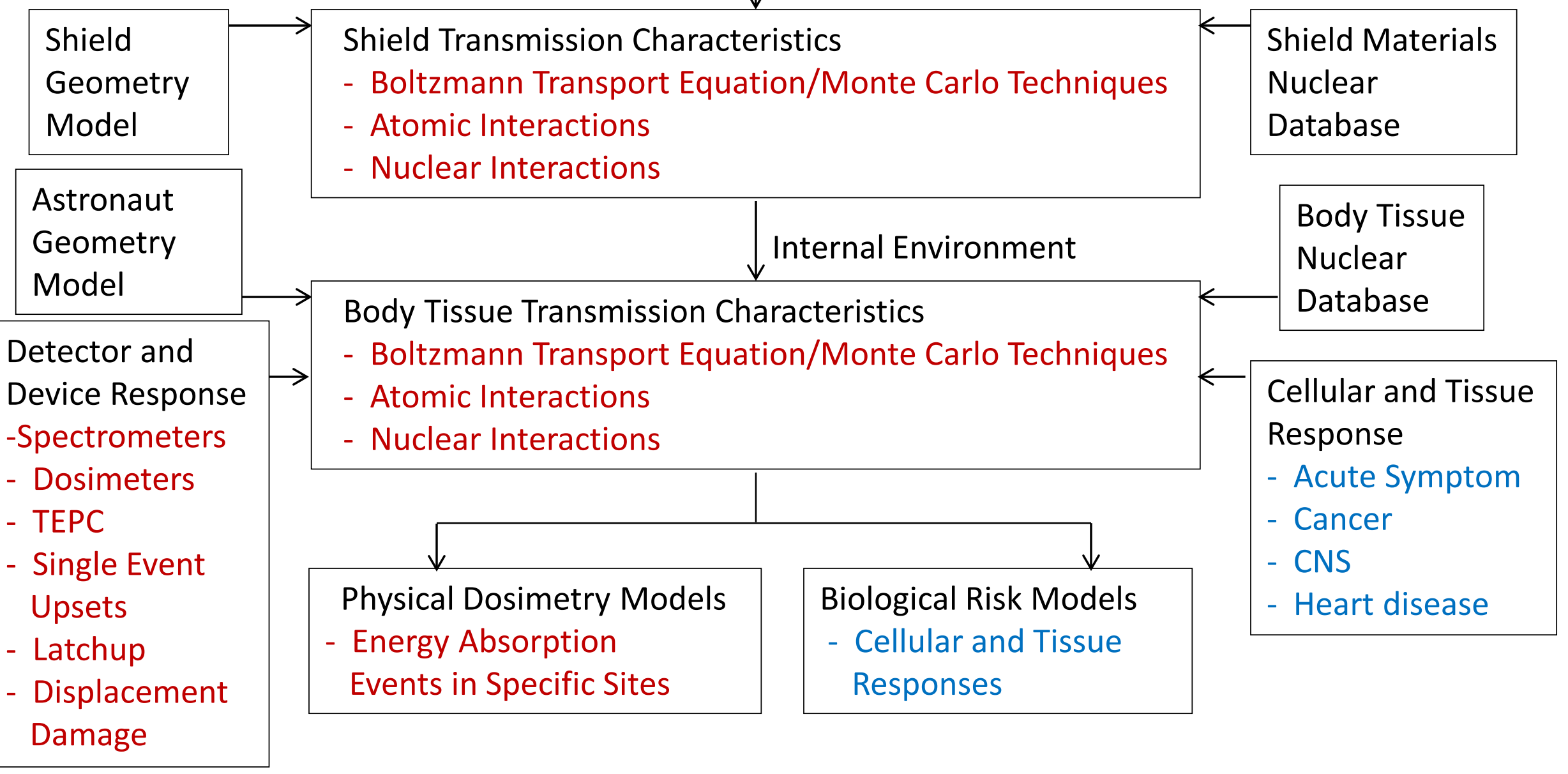




\section{NSRL for Biophysics Applications}

\section{Approximate Composition}

$$
\mathrm{N}_{101.7} \mathrm{O}_{33.1} \mathrm{Al}_{36}
$$

Density: $0.00194 \mathrm{~g} / \mathrm{cm}^{3}$

Thickness: $1.2166 \mathrm{~g} / \mathrm{cm}^{2}$

$\mathrm{N}: 2.0910^{22}$ atoms/g

O: $6.8110^{21}$ atoms/g

Al: $7.4110^{21}$ atoms/g

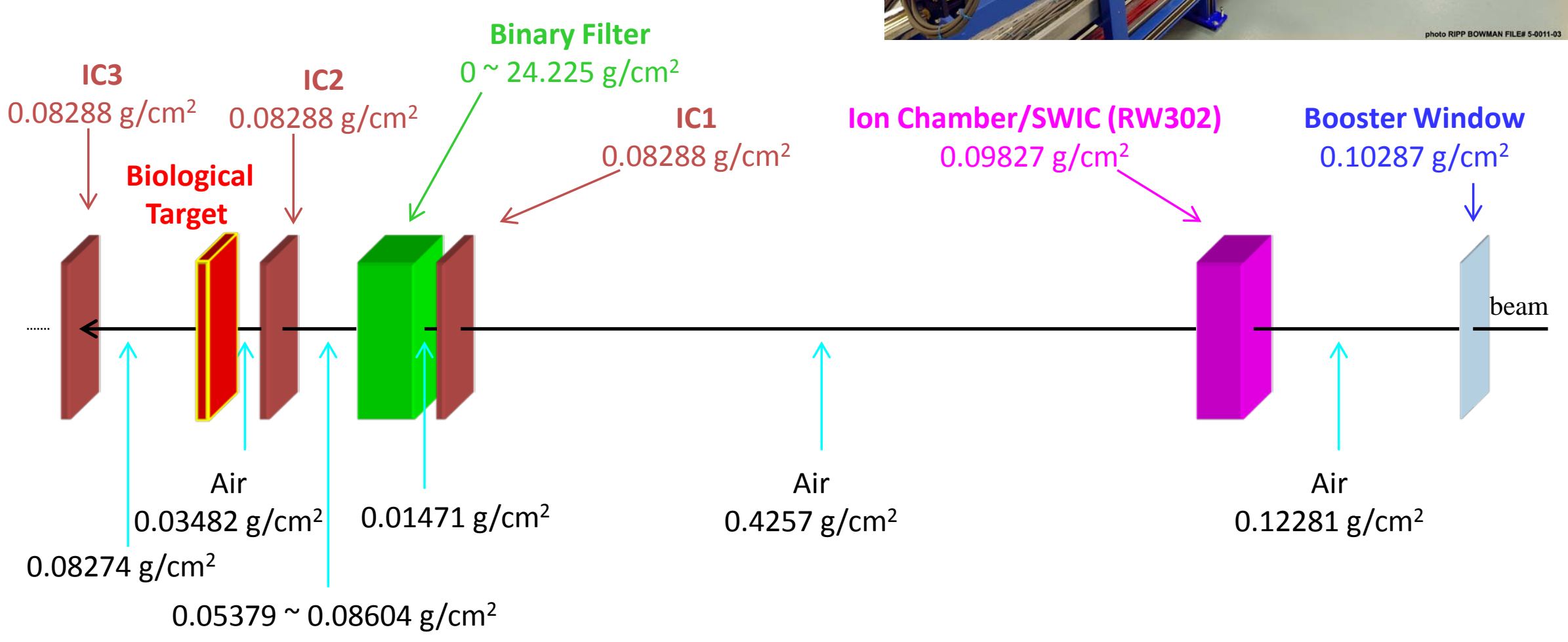




\section{Heavy Ion Reactions}

Abrasion=projectile-target overlap ( $n, p$, and cluster knock-out) Ablation = pre-fragment decay ( $n, p, d, t, h$, alphas de-excitation) Coalescence $=p$ and $n$ knockout form bound states in couple phase space

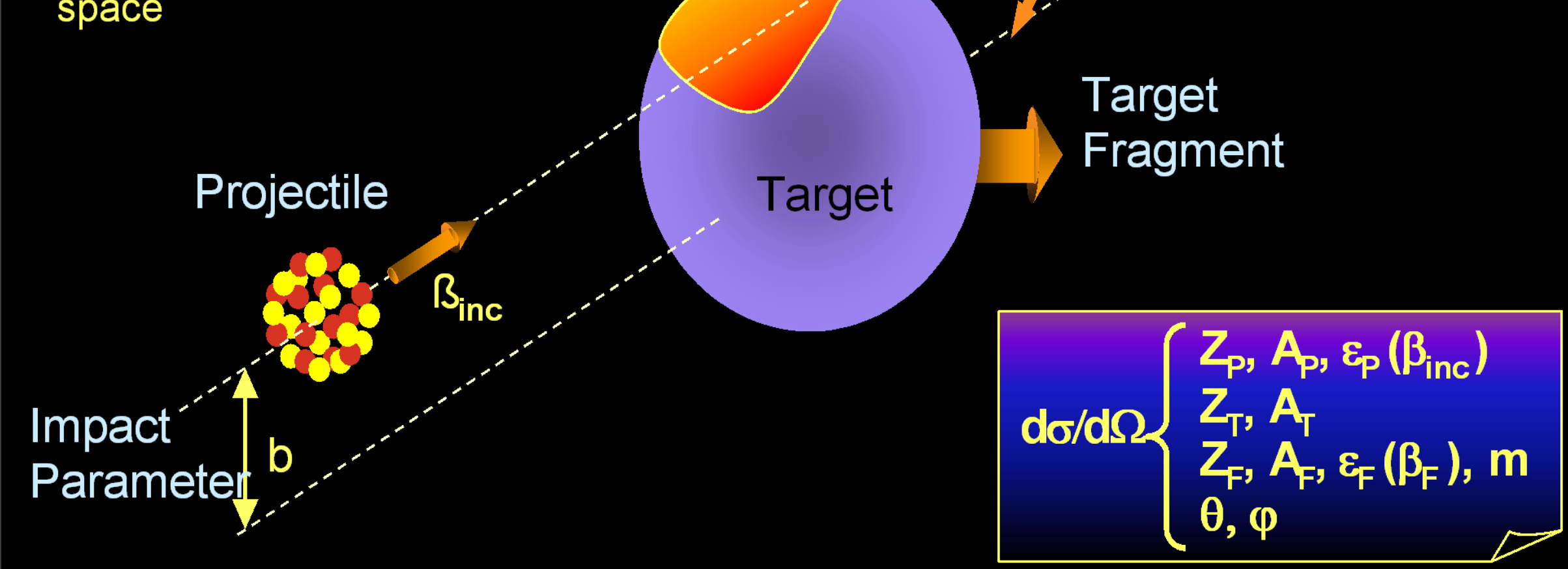




\section{Fragmentation Cross Sections: Comparison of QMSFRG to Si and Fe Beams}
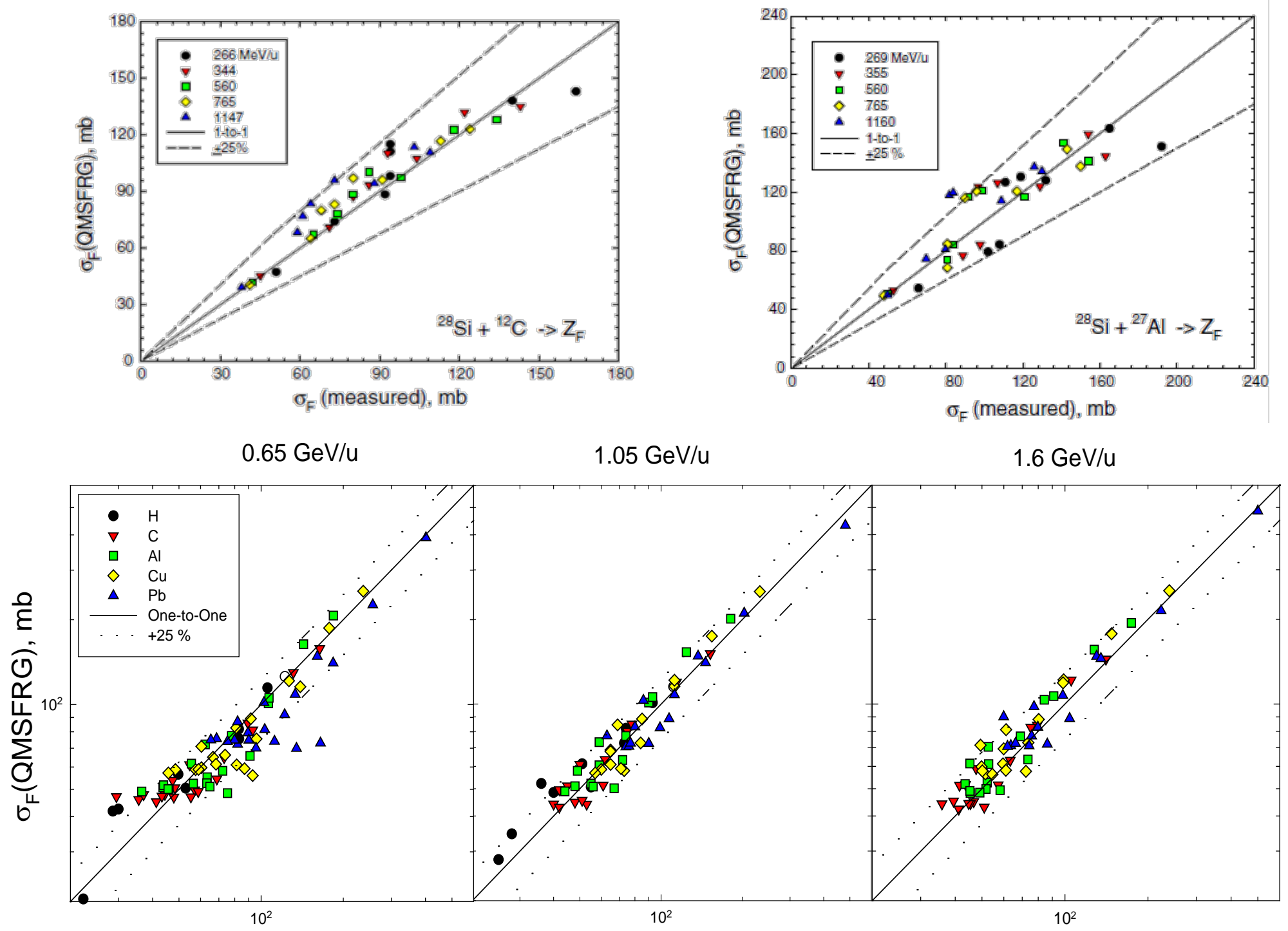

$\sigma_{\mathrm{F}}$ (measured), mb

$\sigma_{F}$ (measured), mb

$\sigma_{\mathrm{F}}$ (measured), mb 


\section{NSRL Bragg Curve Comparison to GCR Event-based Risk Model (GERM)}
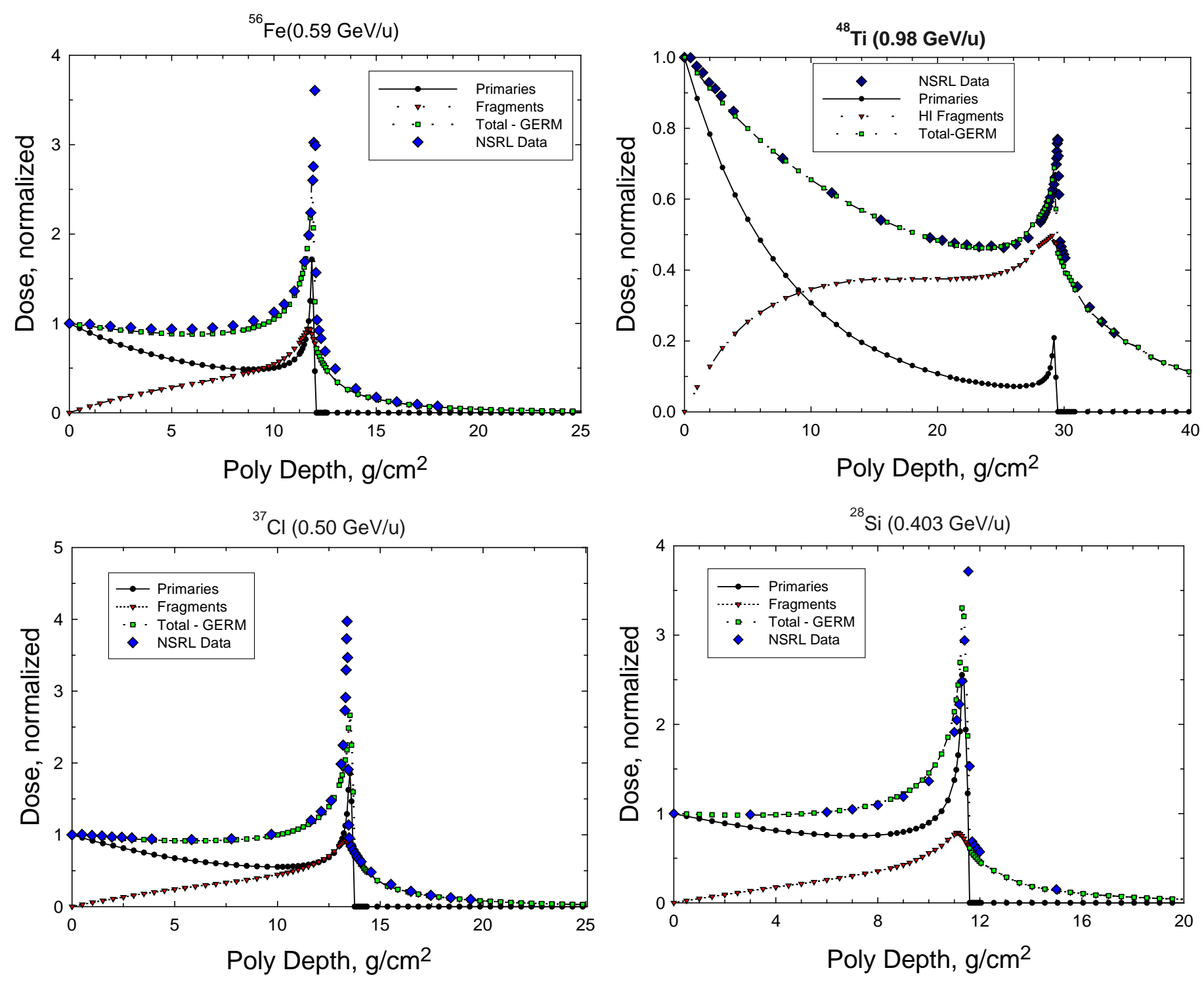

Cucinotta FA et al., Radiat Prot Dosimetry, 2011 


\section{Thick Target Comparison with NASA's GERMCode* and GRNTRN Code*}
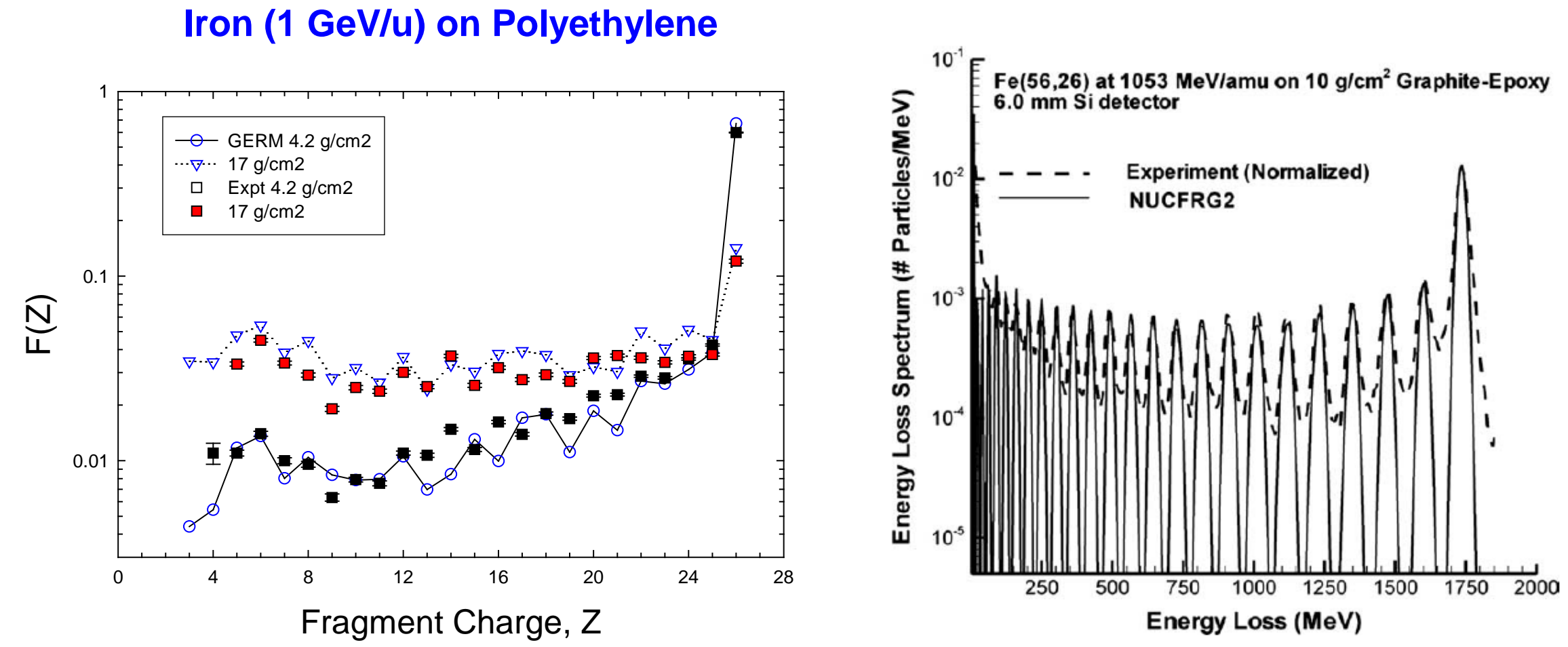

*HZETRN uses identical Nuclear Cross Sections and Atomic Data 


\section{Space Weather Prediction Center, NWS, NOAA}

NOAAISWPC
Boulder, CO 12BiT

$1 \times 1$

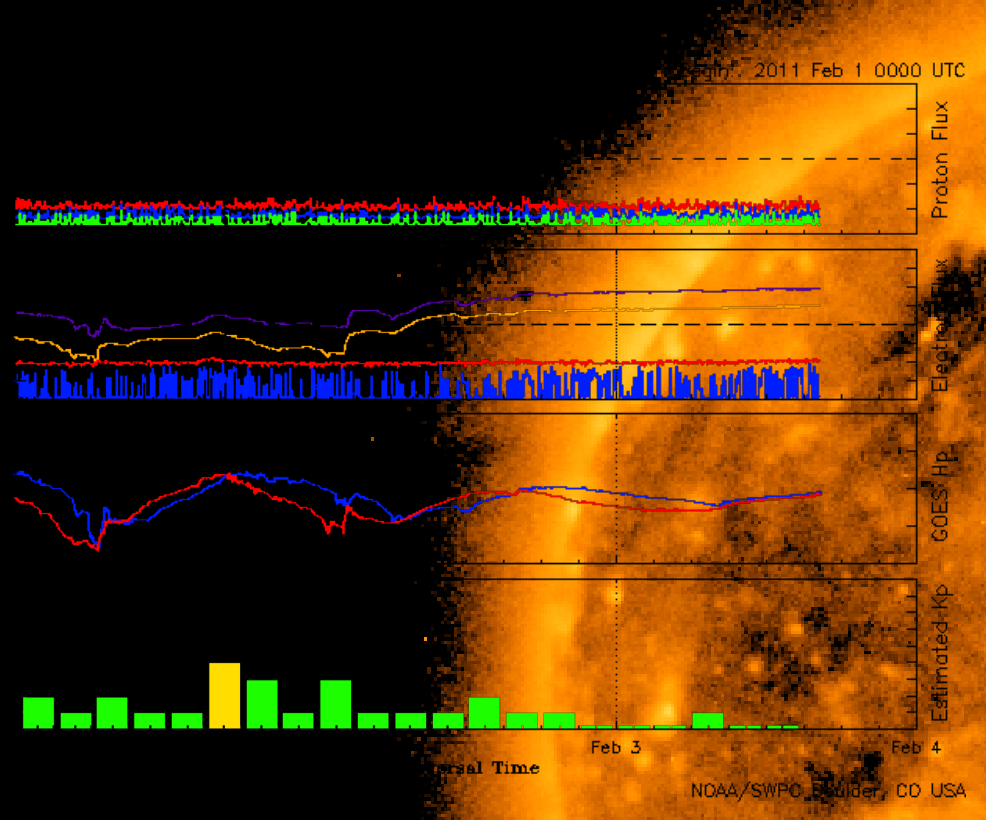

Satellite Environment

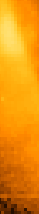

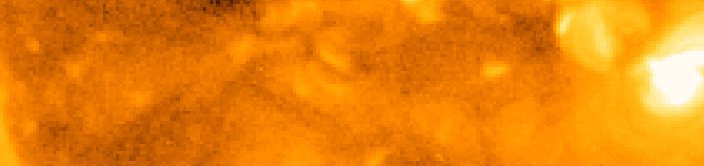

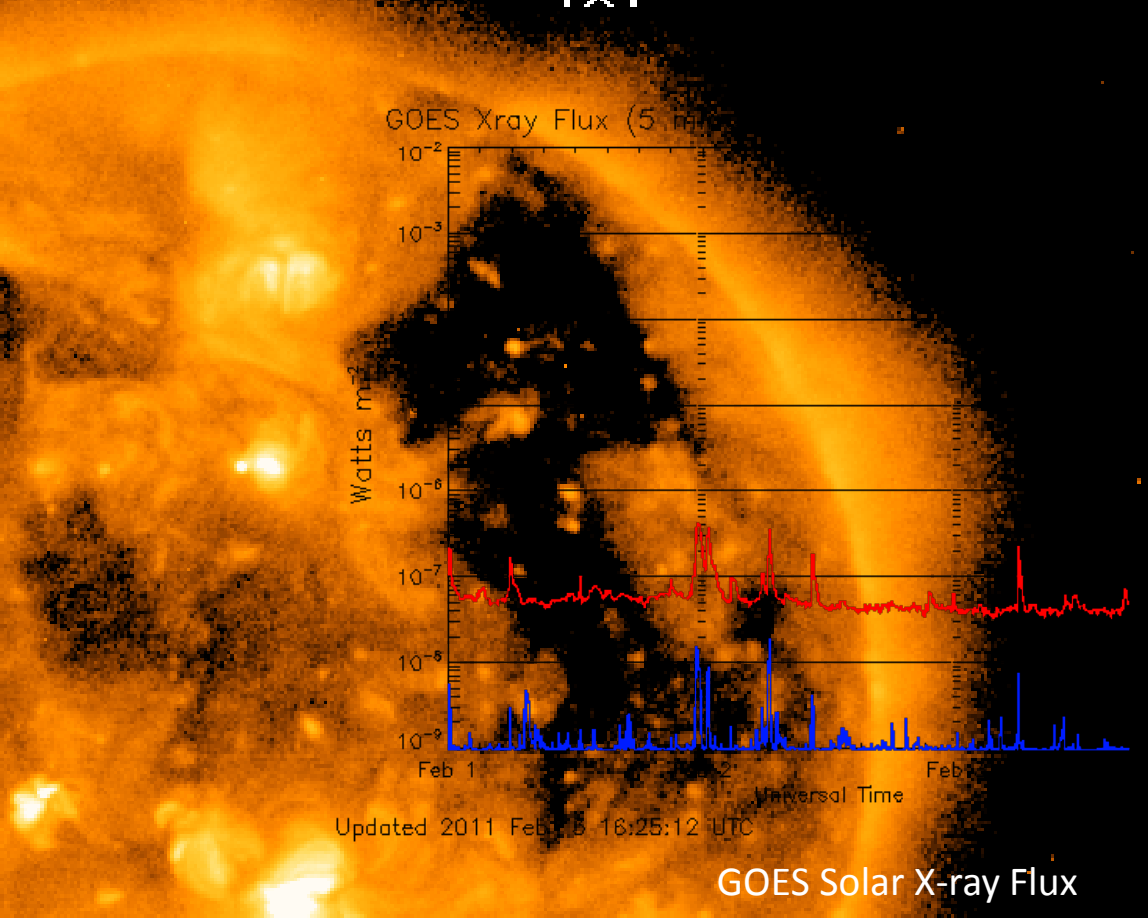

NOAA Scales Activity Range 1 (minor) to 5 (extreme)

NOAA Scale Geomagnetic Storms Solar Radiation Storms Radio Blackouts
Past-24 hours

none none wit? none
Current none none none 


\section{Space Environmental Models}

Fit to Proton Measurements for Continuous Spectrum

Functional Forms with Measurements

- Exponential in Rigidity or Energy: $\Phi(>R)=J_{0} \exp \left(-R / R_{0}\right)$ or $\Phi(>E)=J_{0} \exp \left(-E / E_{0}\right)$

- Sum of Two Exponentials : $\Phi(>E)=J_{1} \exp \left(-E / E_{1}\right)+J_{2} \exp \left(-E / E_{2}\right)$

- Weibull Function in Energy : $\Phi(>E)=J_{0} \exp \left(-\kappa E^{\alpha}\right)$

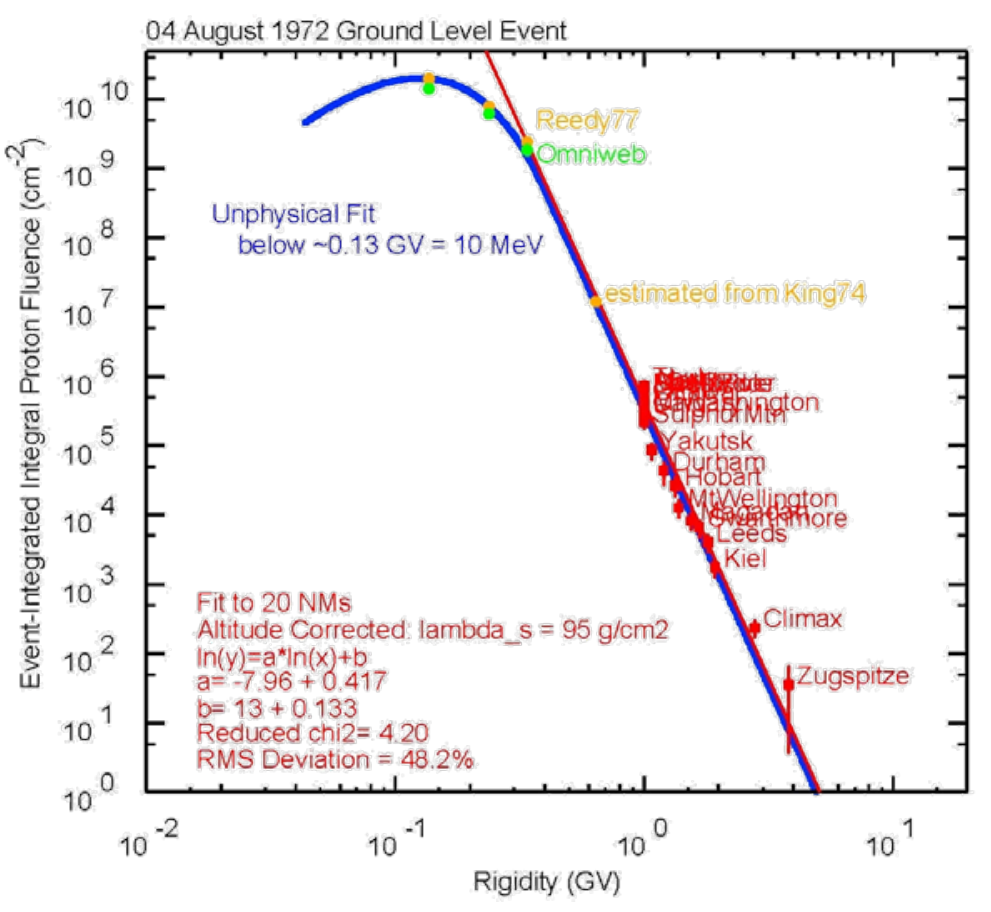

Band Function with 4 Parameters $\left(J_{0}, \gamma_{1}, \gamma_{2}, R_{0}\right)$ : Double Power Law in Rigidity

$\Phi(>R)=J_{0} R^{-\gamma_{1}} e^{-R / R_{0}}$

for $R \leq\left(\gamma_{2}-\gamma_{1}\right) R_{0}$

$\Phi(>R)=J_{0} R^{-\gamma_{2}}\left\{\left[\left(\gamma_{2}-\gamma_{1}\right) R_{0}\right]^{\left(\gamma_{2}-\gamma_{1}\right)} e^{\left(\gamma_{1}-\gamma_{2}\right)}\right\} \quad$ for $R \geq\left(\gamma_{2}-\gamma_{1}\right) R_{0}$

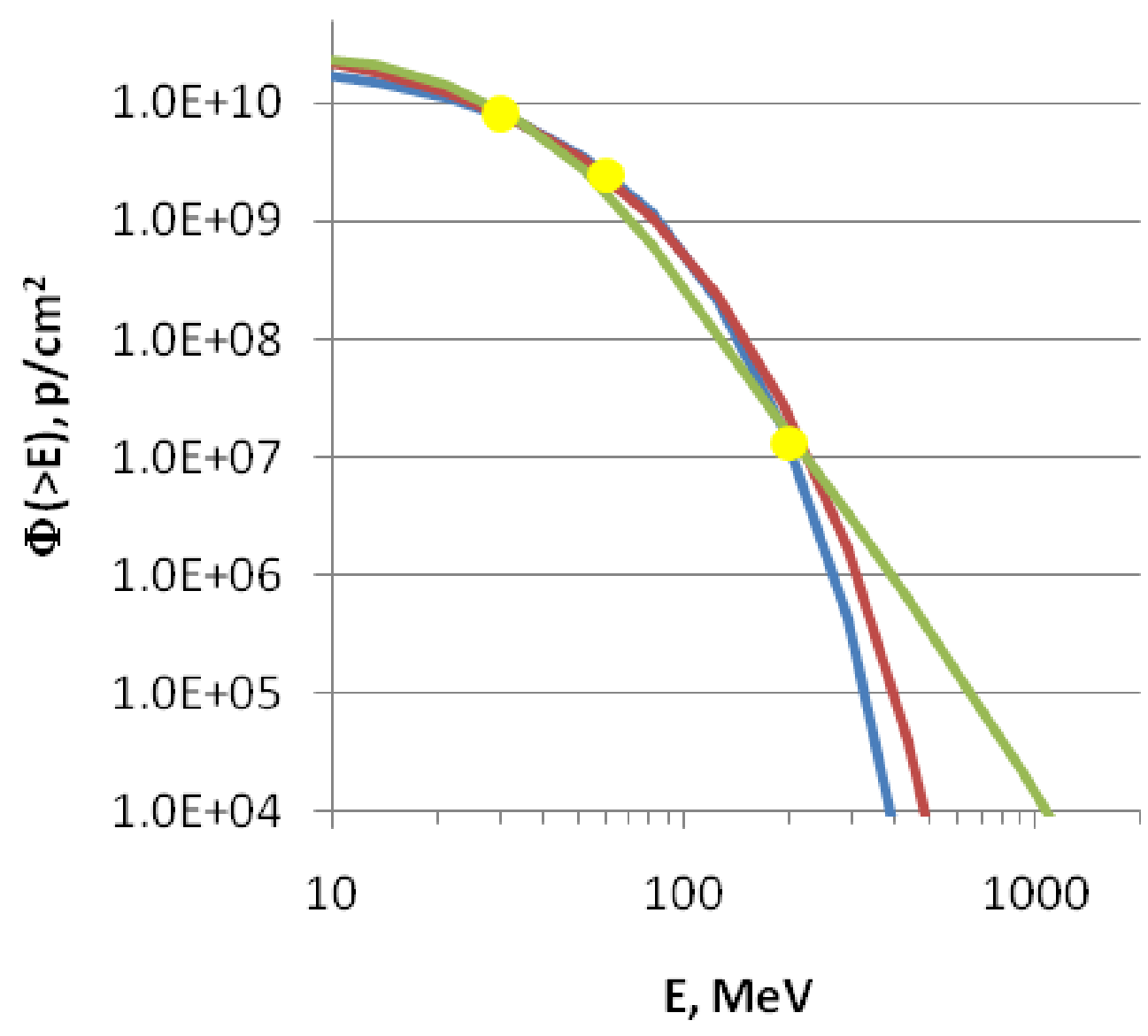




\section{Interplanetary Galactic Cosmic Ray Energy Spectra}

\section{Advanced Composition Explorer/Cosmic Ray Isotope Spectrometer}

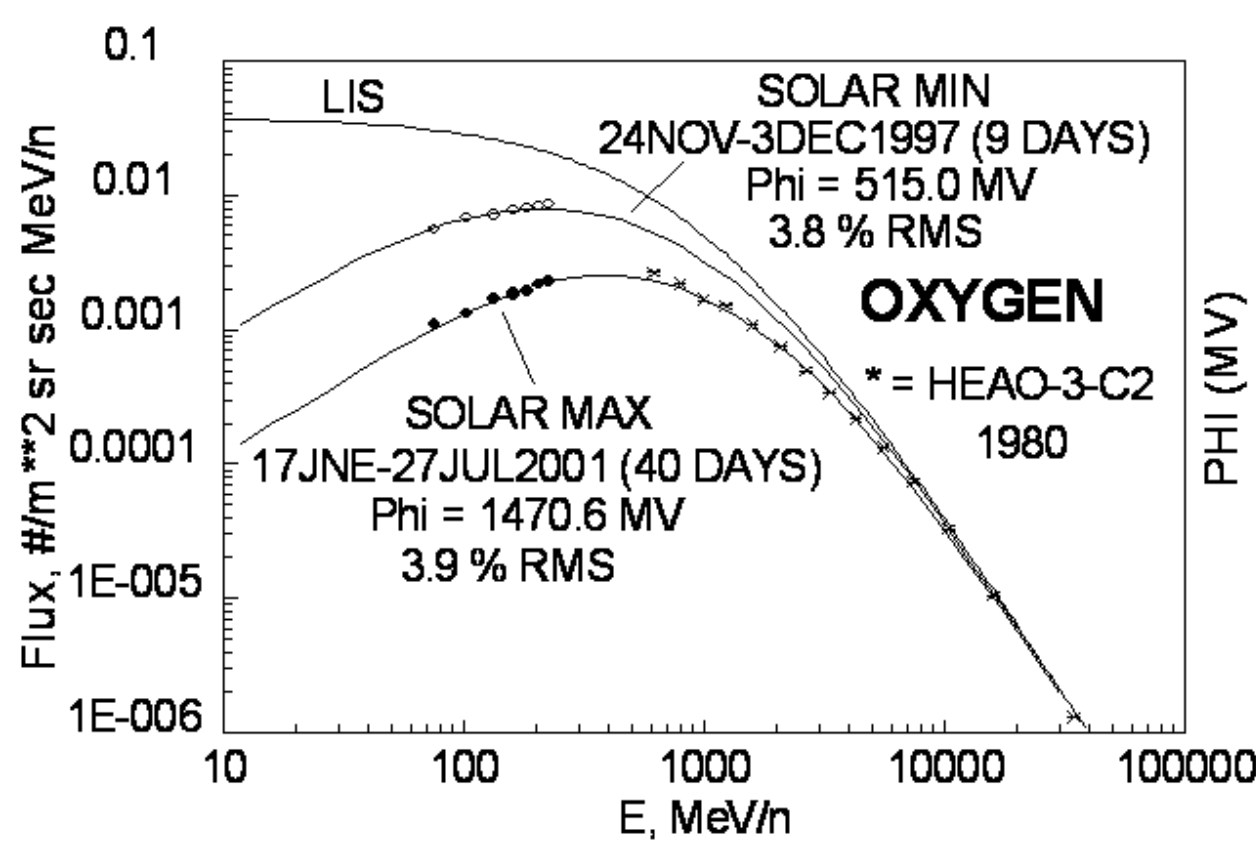

Badhwar-O'Neill Model fit of ACE CRIS oxygen energy spectra measurements near solar minimum and near solar maximum

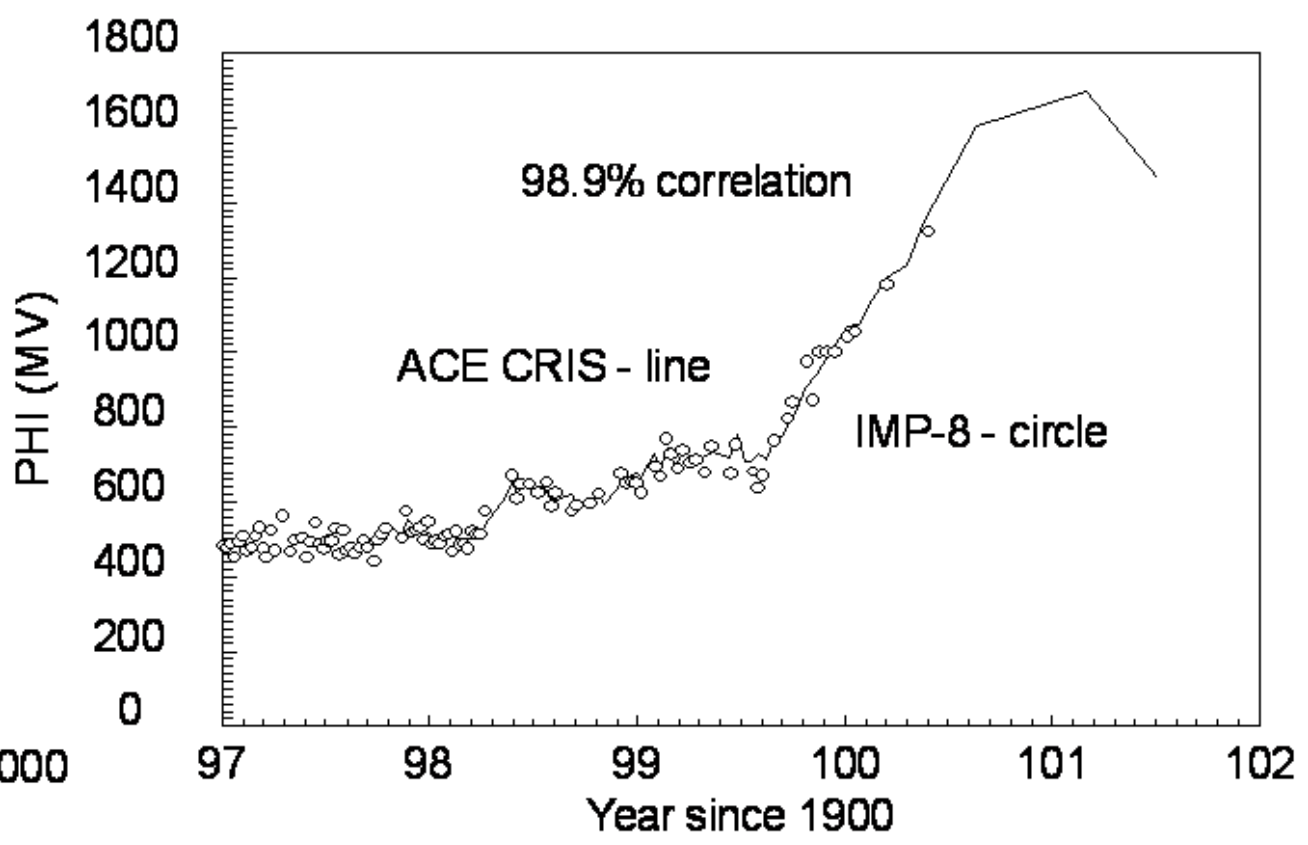

Solar modulation parameter:

ACE CRIS oxygen measurements (line); IMP-8 (Z>8) channel 7 measurements $(0)$ 


\section{Inter-Comparisons of Transport Codes}
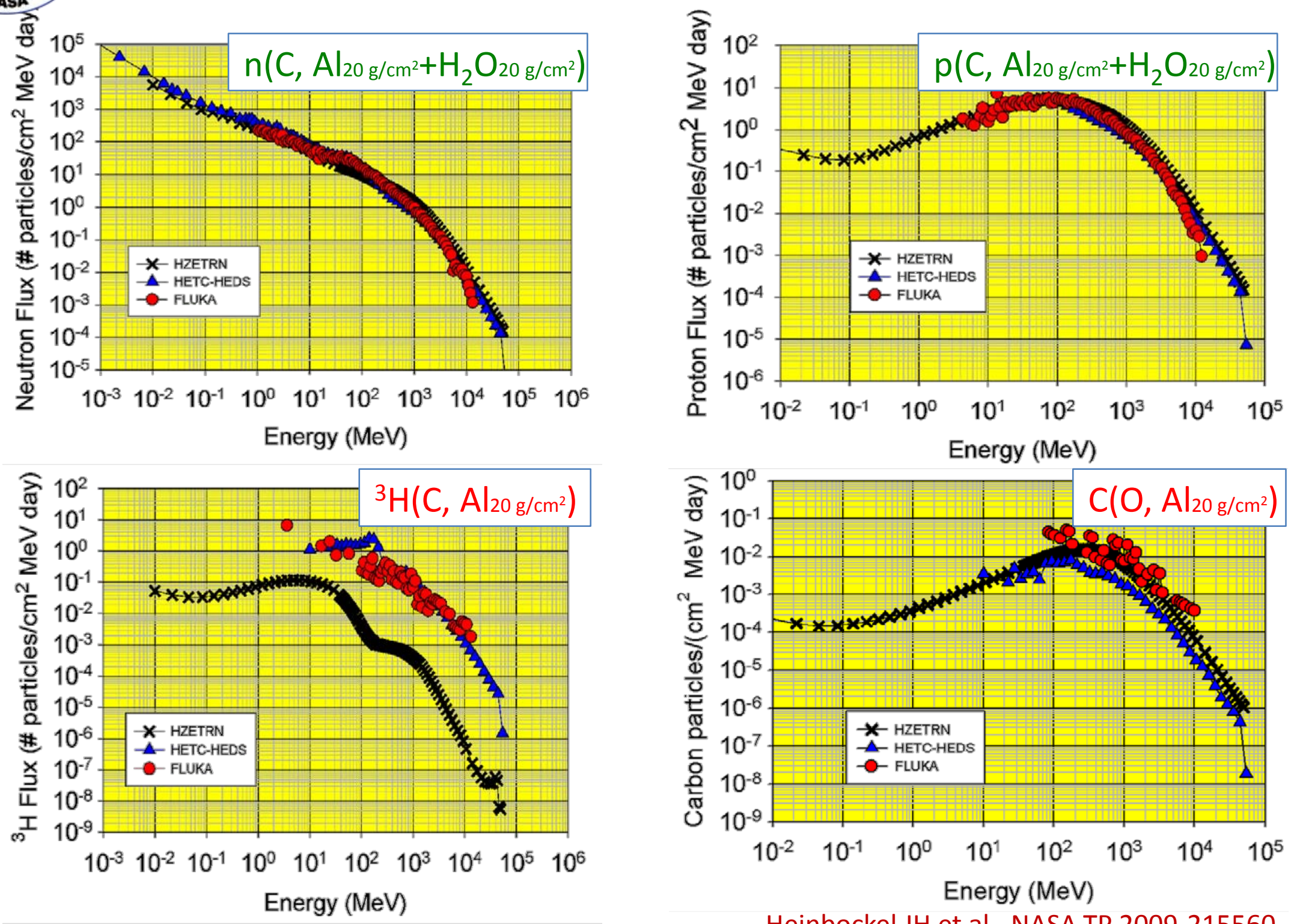

Heinbockel JH et al., NASA TP 2009-215560, 2009 


\section{Comparisons with Flight Measurements}
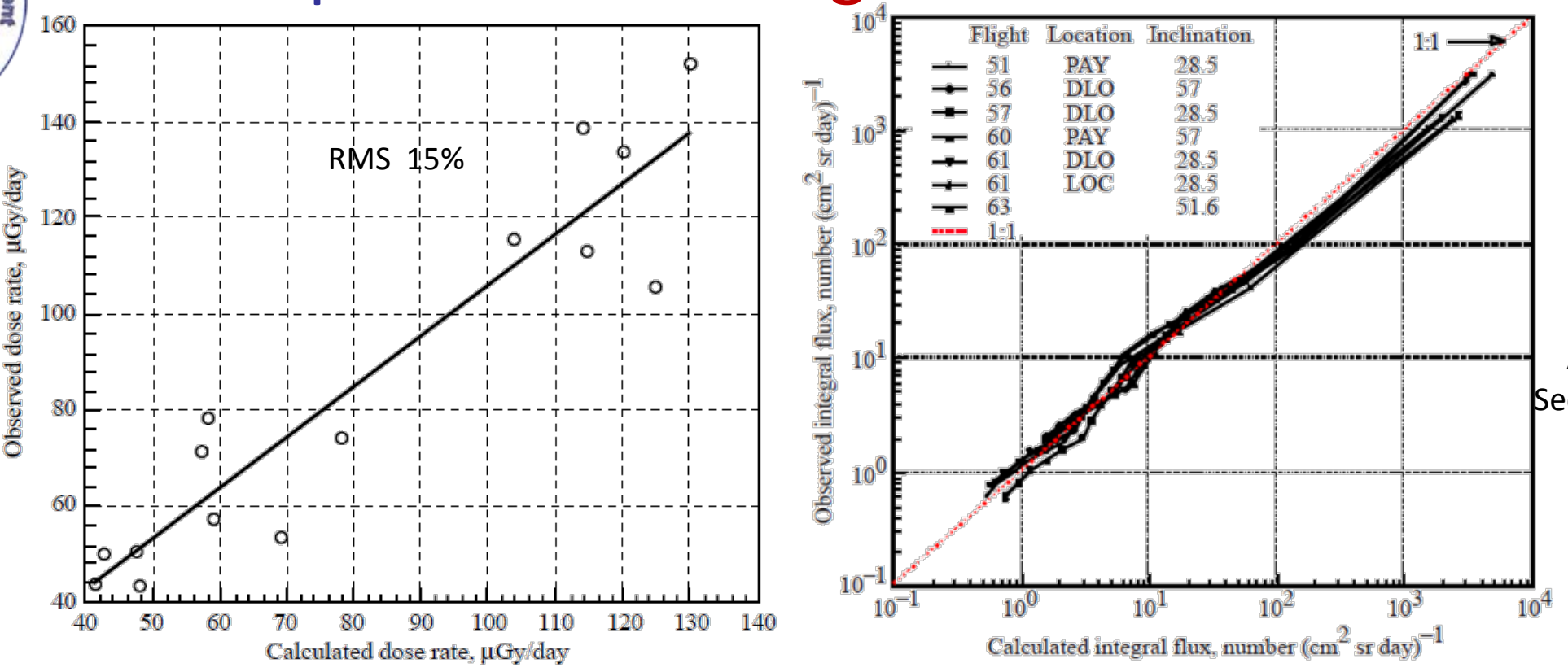

$1.5-2.7 X$

Albedo protons Albedo neutrons econdary neutron

Geomagnetic

tansmission function
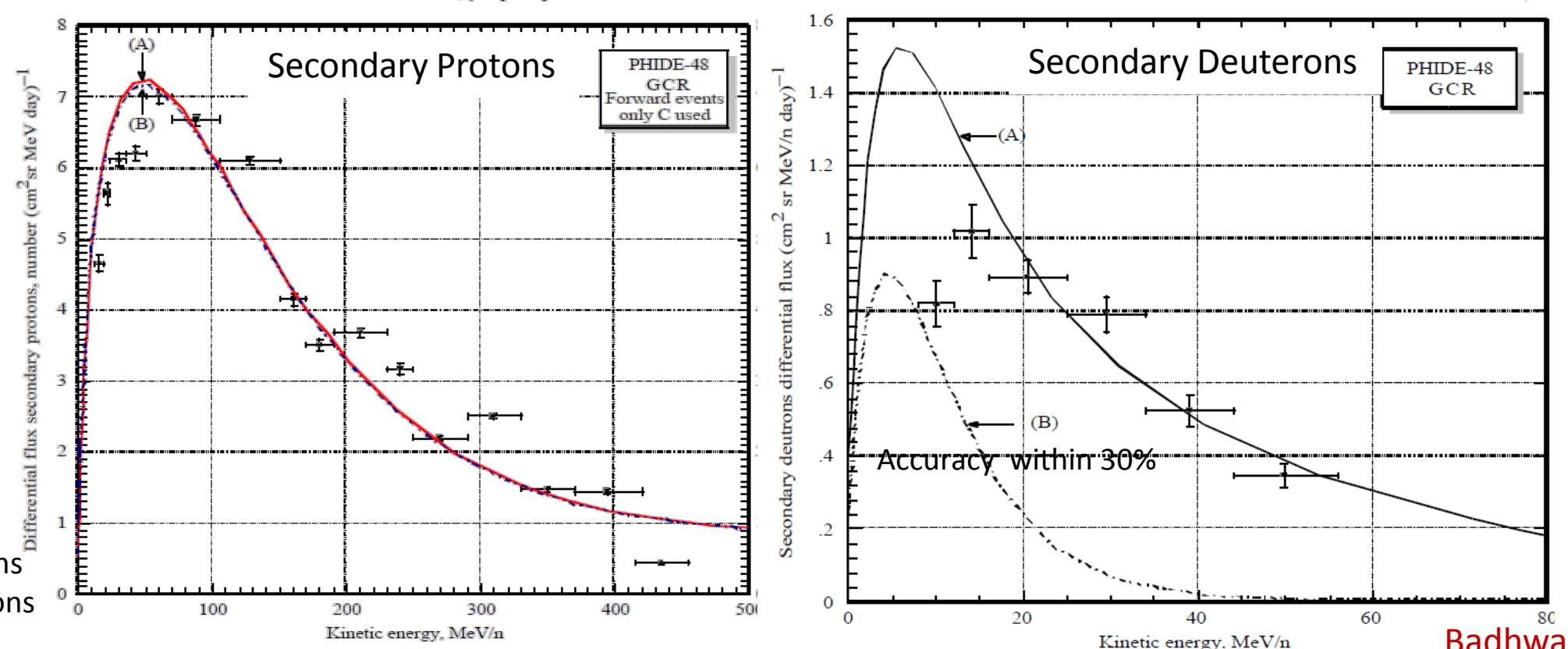

Badhwar GD, 1997 


\section{Evaluation of Detector Response}

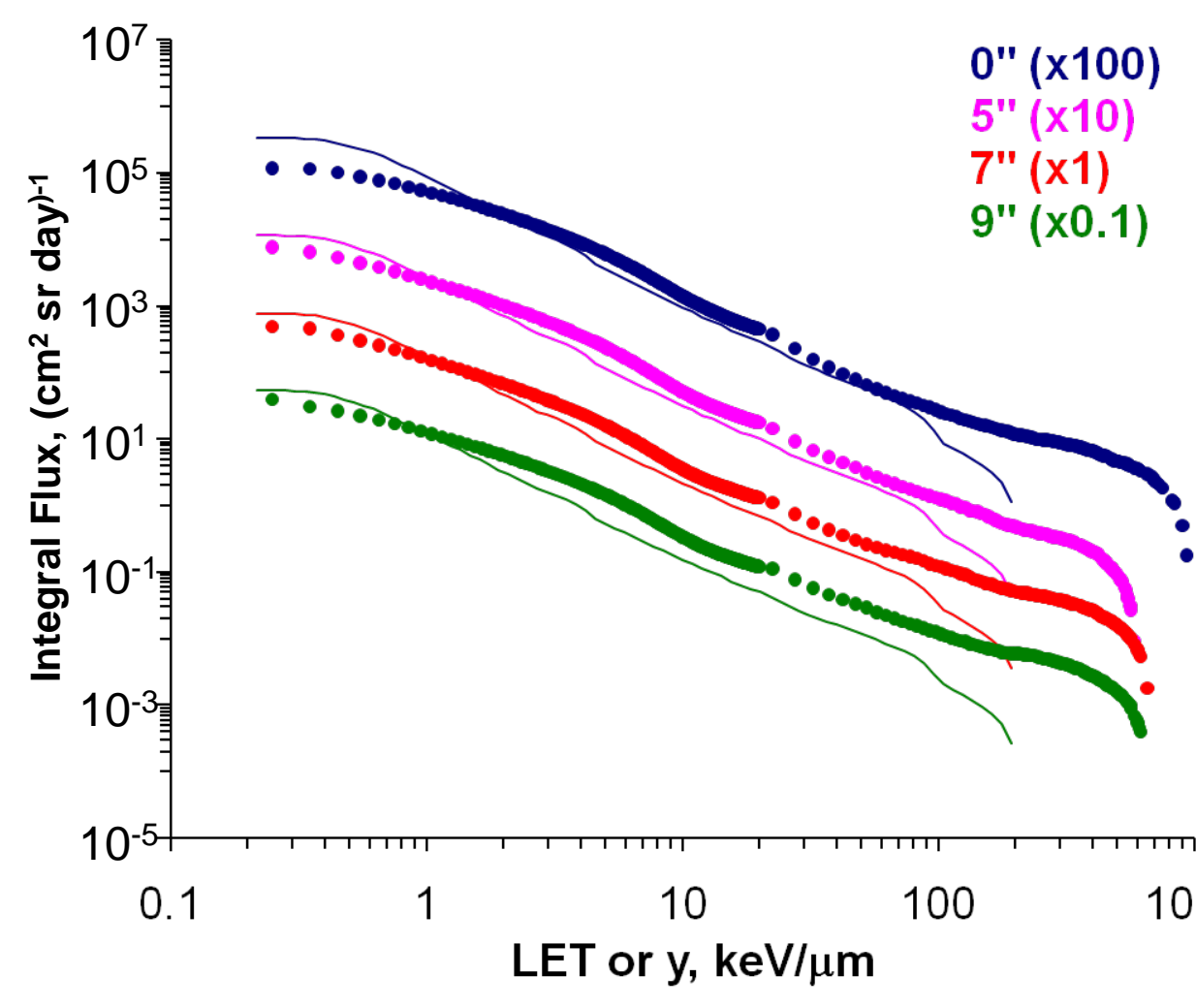

without TEPC response

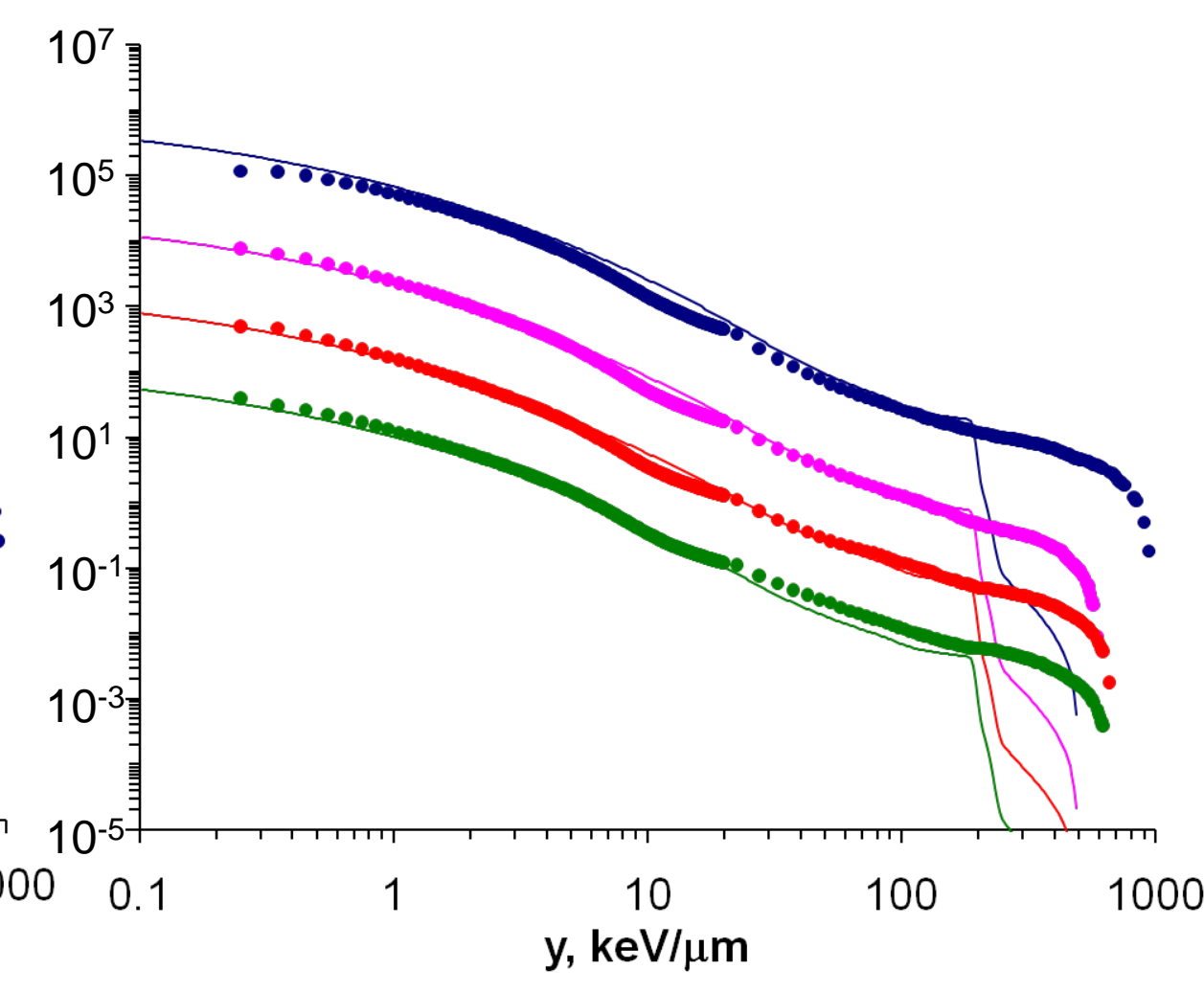

with TEPC response 


\section{Phantom Torso Experiment (PTE) of ISS/STS TLD Dose Contours of Brain Slice}
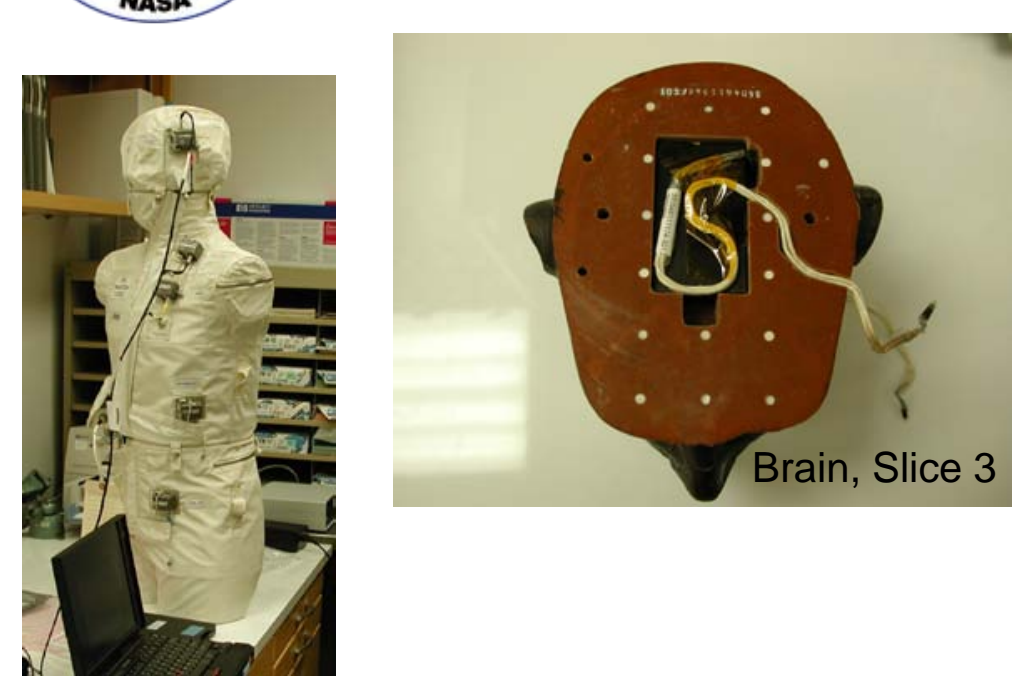

TLD Type

- 100

- 300 600

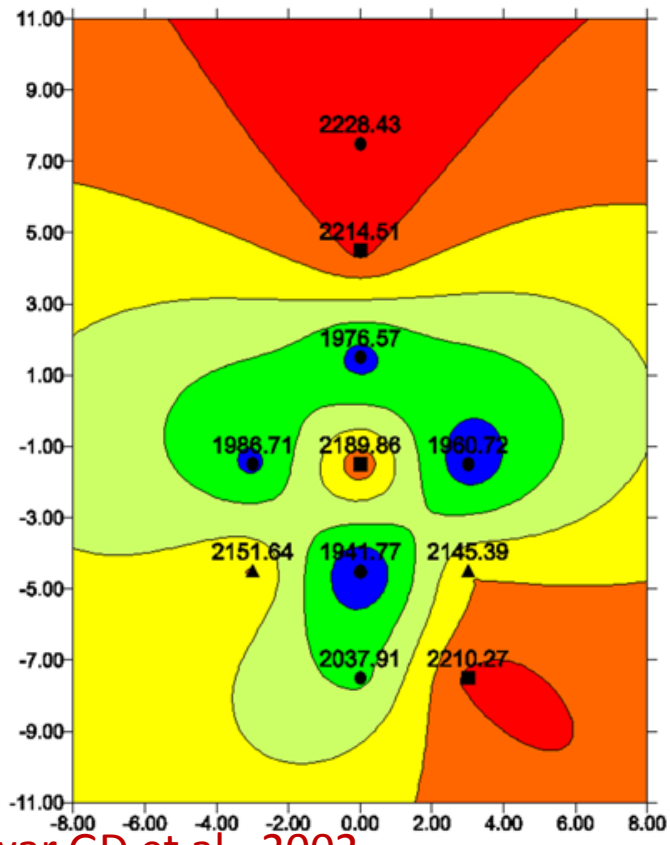

Badhwar GD et â., 2002
Organ Dose Equivalent using CR-39/TLD, mSv

\begin{tabular}{|l|c|c|c|}
\multicolumn{5}{|c}{ Organ Dose Equivalent using CR-39/TLD, mSv } \\
\hline Tissue & Measured & HZETRN/QMSFRG & Difference (\%) \\
\hline Skin & $4.5 \pm 0.05$ & 4.7 & 4.4 \\
\hline Thyroid & $4.0 \pm 0.21$ & 4.0 & 0 \\
\hline Bone surface & $5.2 \pm 0.22$ & 4.0 & -23.1 \\
\hline Esophagus & $3.4 \pm 0.49$ & 3.7 & 8.8 \\
\hline Lung & $4.4 \pm 0.76$ & 3.8 & -13.6 \\
\hline Stomach & $4.3 \pm 0.94$ & 3.6 & -16.3 \\
\hline Liver & $4.0 \pm 0.51$ & 3.7 & -7.5 \\
\hline Bone marrow & $3.4 \pm 0.40$ & 3.9 & 14.7 \\
Colon & $3.6 \pm 0.42$ & 3.9 & 8.3 \\
\hline Bladder & $3.6 \pm 0.24$ & 3.5 & -2.8 \\
\hline Gonad & $4.7 \pm 0.71$ & 3.9 & -17.0 \\
Chest & $4.5 \pm 0.11$ & 4.5 & 0 \\
\hline Remainder & $4.0 \pm 0.57$ & 4.0 & 0 \\
\hline Effective dose & $4.1 \pm 0.22$ & 3.9 & -4.9 \\
\hline
\end{tabular}

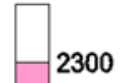

Yasuda et al., 2002

Active Dosimetry Data, mGy/d

\begin{tabular}{c|c|c|c|c|c|c|c|c|}
\hline Organ & \multicolumn{2}{|c|}{ Trapped } & \multicolumn{2}{|c|}{ GCR } & \multicolumn{2}{c|}{ Total } & Difference \\
\hline & Expt & Model & Expt & Model & Expt & Model & $(\%)$ \\
\hline Brain & 0.051 & 0.066 & 0.076 & 0.077 & 0.127 & 0.143 & 13.3 \\
Thyroid & 0.062 & 0.072 & 0.074 & 0.077 & 0.136 & 0.148 & 9.4 \\
Heart & 0.054 & 0.061 & 0.075 & 0.076 & 0.129 & 0.137 & 6.7 \\
Stomach & 0.050 & 0.057 & 0.076 & 0.077 & 0.126 & 0.133 & 5.5 \\
Colon & 0.055 & 0.056 & 0.073 & 0.076 & 0.128 & 0.131 & 2.5 \\
\hline
\end{tabular}

Cucinotta FA et al., 2008 


\section{Predictions for Mars Mission}

1-y interplanetary space $\quad$ 1-y Mars surface $\quad$ 30-month Mars mission

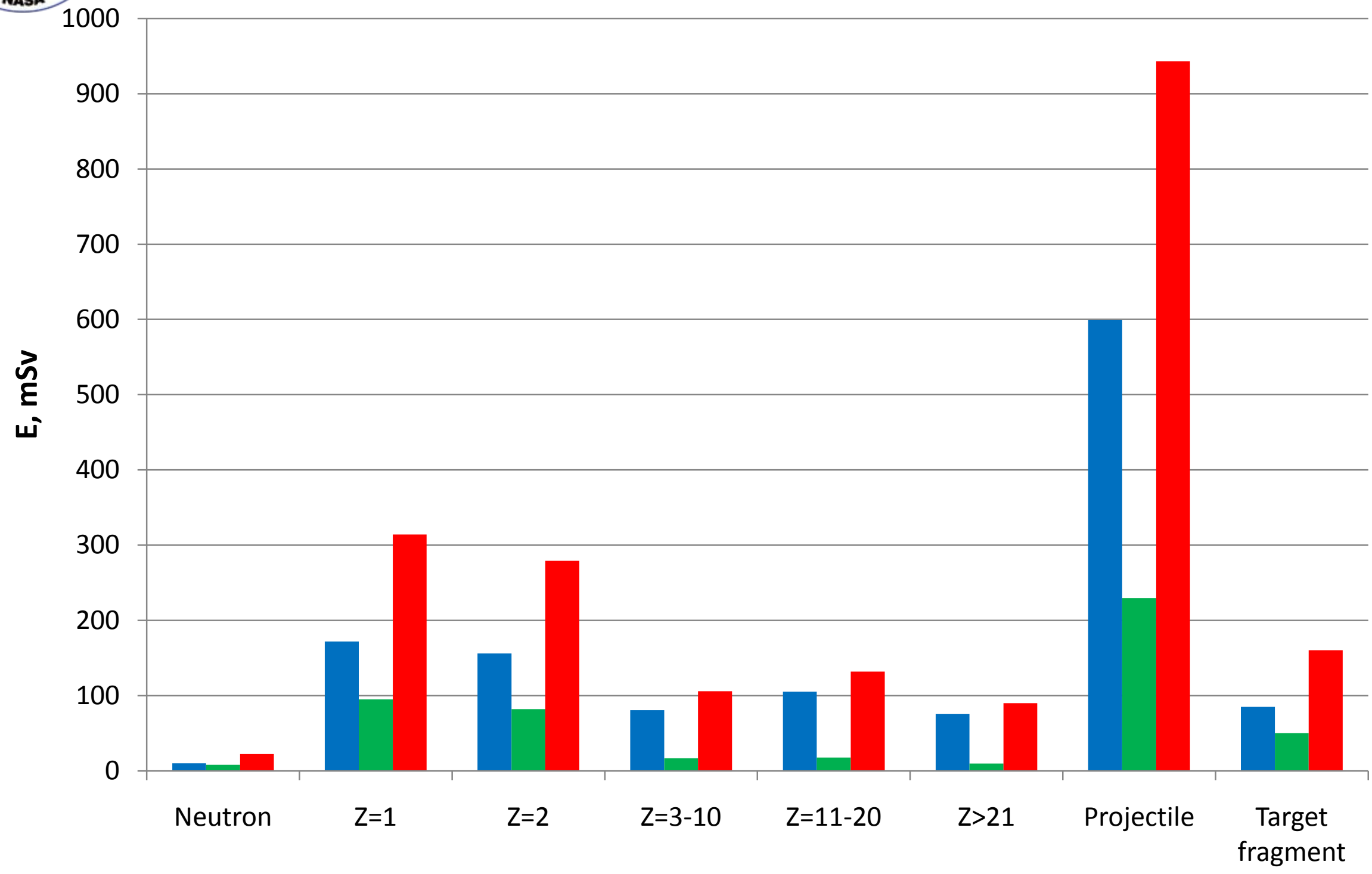

Radiation type 


\section{Annual Effective Dose for Male}

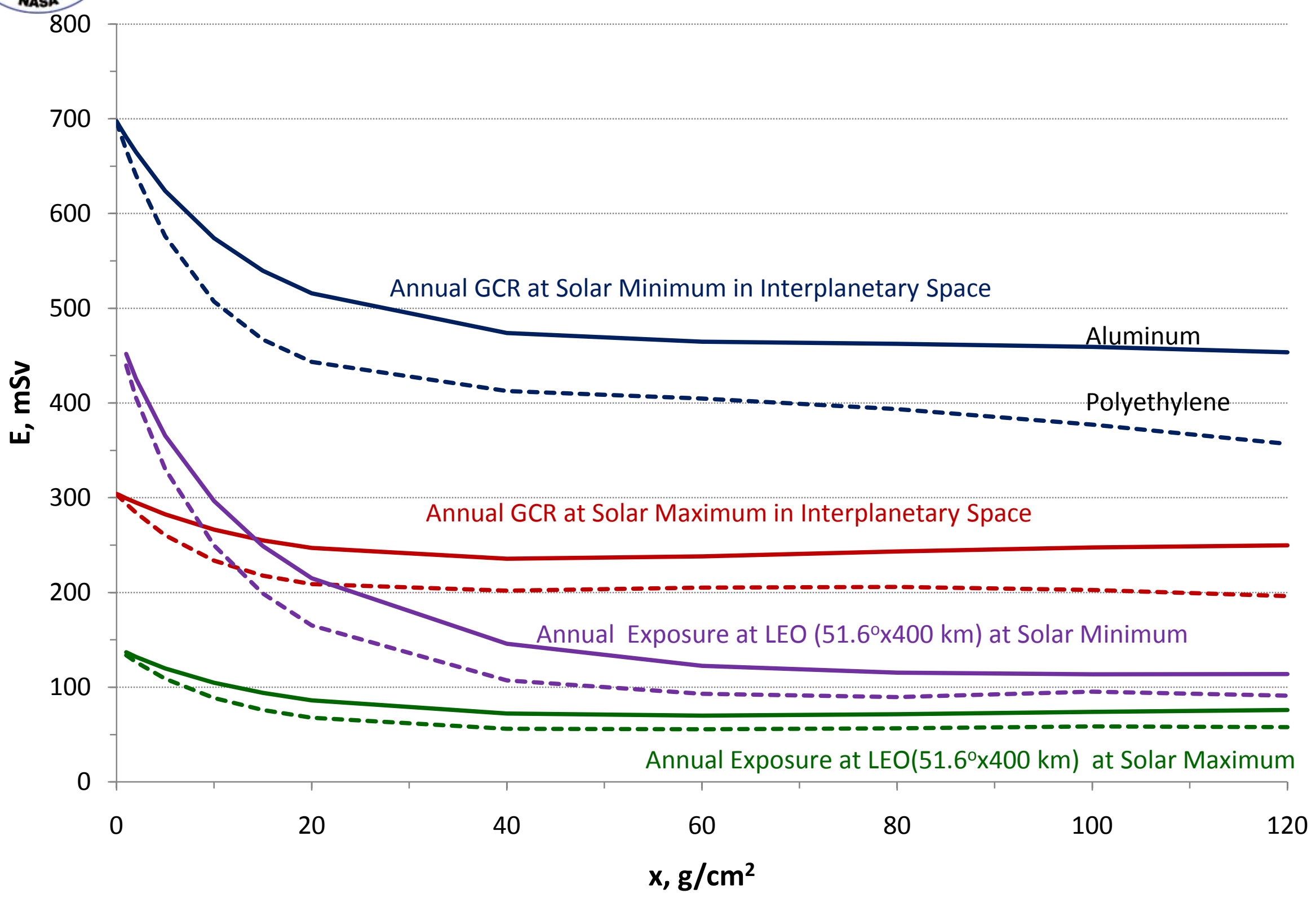




\section{Model-based Prediction of SPE Occurrence}
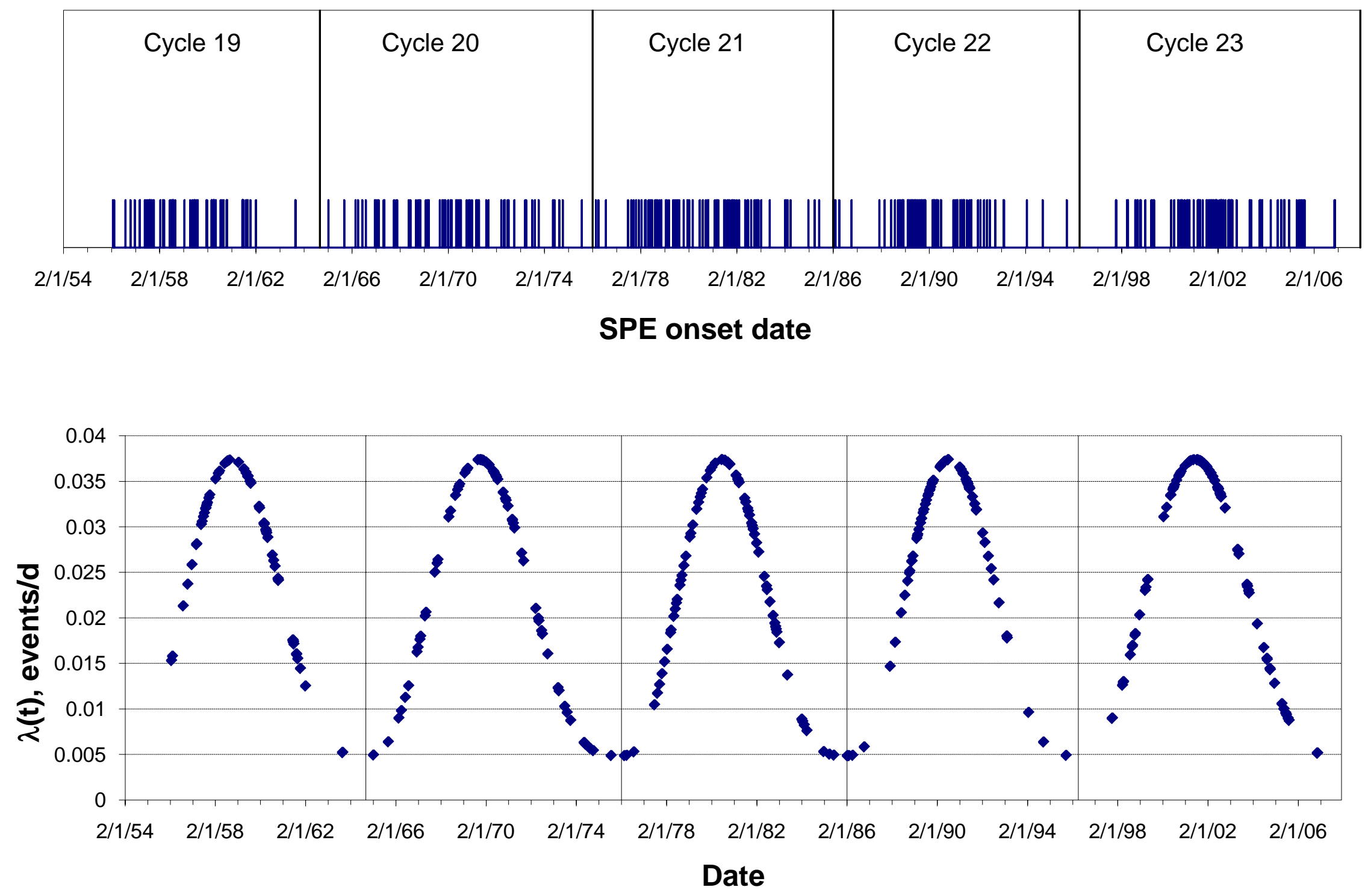


\section{Model-based Prediction of SPE Fluence}

Propensity of SPEs: Hazard Function of Offset $\beta$ Distribution Density Function

$$
\lambda(t)=\frac{\lambda_{0}}{4000}+\frac{K}{4000} \frac{\Gamma(p+q)}{\Gamma(p) \Gamma(q)}\left(\frac{t}{4000}\right)^{p-1}\left(1-\frac{t}{4000}\right)^{q-1} \quad(0 \leq t \leq 4000)
$$

95 percentile

^ 25 percentile
+90 percentile

* 10 percentile $\times 75$ percentile

- 5 percentile
- Median

-- The Carrington Event

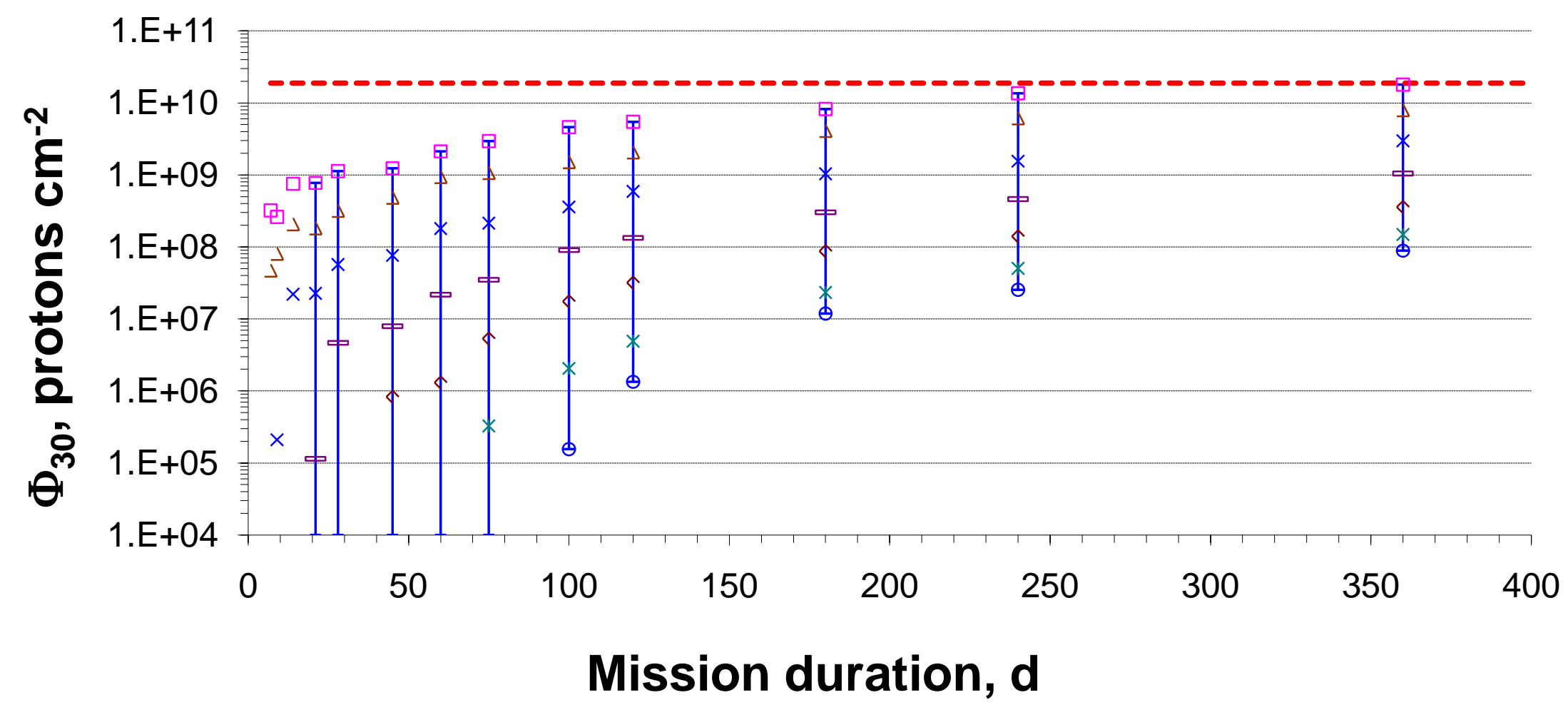




\section{Effective dose on Mars Surface with MOLA Topography}

\begin{tabular}{|c|c|c|c|c|}
\hline \multirow{2}{*}{$\begin{array}{l}\text { Altitude, } \\
\text { km }\end{array}$} & \multirow{2}{*}{$\mathrm{T},{ }^{\circ} \mathrm{C}$} & \multirow{2}{*}{$\mathbf{p}, \mathbf{k P a}$} & \multicolumn{2}{|c|}{$\begin{array}{l}\text { Atmospheric shielding } \\
\text { thickness, } \mathrm{g} / \mathrm{cm}^{2}\end{array}$} \\
\hline & & & $\begin{array}{l}\text { Low density } \\
\text { model }\end{array}$ & $\begin{array}{l}\text { High density } \\
\text { model }\end{array}$ \\
\hline 8.0 & -41.16 & 0.34 & 0.14 & 0.19 \\
\hline 4.0 & -34.99 & 0.49 & 6.73 & 9.25 \\
\hline 2.0 & -33.00 & 0.58 & 10.97 & 15.08 \\
\hline 0.0 & -31.00 & 0.7 & 16.00 & 22.00 \\
\hline-2.0 & -29.00 & 0.84 & 19.04 & 26.17 \\
\hline-4.0 & -27.01 & 1.00 & 22.64 & 31.13 \\
\hline-8.0 & -23.02 & 1.44 & 32.00 & 44.00 \\
\hline
\end{tabular}
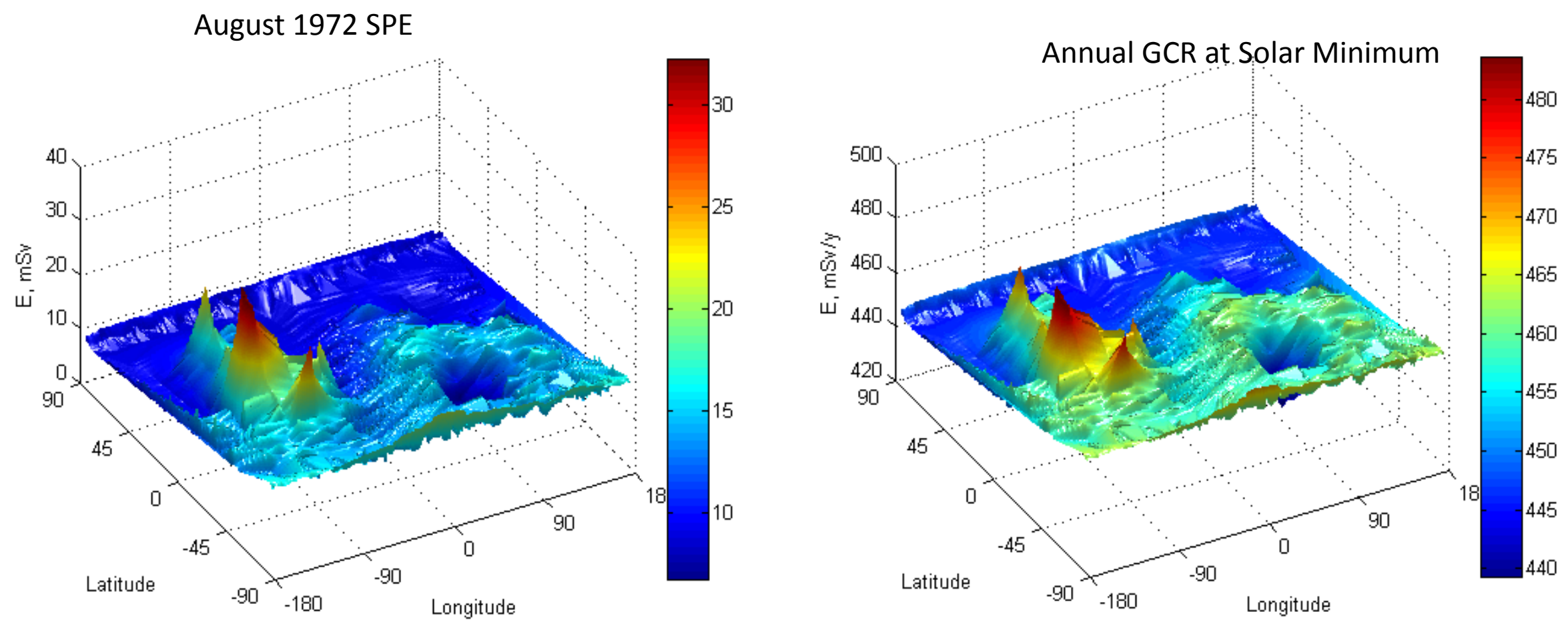


\section{Conclusion}

- Highly accurate descriptions of space environment models are available:

$>$ Inter-stellar GCR composition accuracy : $5 \%$ for abundant elements (oxygen, carbon, and iron); less than $10 \%$ for all major GCR components; and solar modulation parameters with the $98.9 \%$ correlation in various spacecraft measurements.

$>$ Probabilistic SPE occurrence model as a tool for managing the risk.

$>$ Comprehensive catalogue of GLE fluences and spectra assembled for shielding design application using satellites and NM spectra;

- Radiation transport codes have been validated extensively:

$>$ QMSFRG model agrees for absorption $\sigma$-section within $+5 \%$ and elemental fragment $\sigma$-section $\pm 25 \%$.

$>$ Good agreement found from inter-comparisons of transport codes.

$>$ Comparison of model prediction to flight measurements: accuracy less than $15 \%$ for GCR dose rates; $\sim 25 \%$ for secondary particles; and $\pm 30 \%$ for quality factors by TEPC.

$>$ Minor scientific questions remained: low-energy light ion cross section, albedo protons, secondary pions, and kaons.

- Space Radiation Shield Design Tool for the reliable and realistic radiation simulation in the early design process of exploration missions:

$>$ Environmental models, shielding and body geometry models, atomic and nuclear interaction and fragmentation models are incorporated. 